\title{
Development of Three-Axis Active Vibration Isolator Using Displacement Cancellation Technique*
}

\author{
Mhia Md. Zaglul SHAHADAT**, Takeshi MIZUNO**, Yuji ISHINO** \\ and Masaya TAKASAKI** \\ ** Department of Mechanical Engineering, Saitama University, \\ Shimo-Okubo 255, Sakuraku, Saitama 338-8570, Japan \\ Email: shahadat230@yahoo.com
}

\begin{abstract}
A three-degree-of-freedom (3-DOF) active vibration isolation system using displacement cancellation control technique is developed. The displacement cancellation concept is presented and implemented in this research to obtain zero compliance of vibration isolation table against direct static force as well as to suppress the dynamic disturbances acting on the isolation table. In the displacement cancellation control technique, relative movements of the vibration free table are followed with I-PD control and movements of the middle mass respect to base are controlled with PD control. The corresponding controllers are designed in such way so that actuator can generate opposite force against disturbance to cancel the effect of displacement of the middle mass on the vibration isolation table, which finally leads zero compliance of the isolation table. Voice coil motors (VCMs) are used for getting linear actuating effect and corresponding displacements of the table are detected by eddy-current gap sensors. The experimental results showed that the developed system with displacement cancellation mode control has capability to maintain zero compliance against direct static force in the 3-DOF motions associated with horizontal translations and a vertical rotation. Moreover experiments have been conducted to measure the dynamic responses of the vibration isolation table to direct disturbance.
\end{abstract}

Key words: Active Control, Vibration Isolation, Displacement Cancellation, Infinite Stiffness, Zero Compliance

\section{Introduction}

The precision vibration isolation plays an important role to meet the current demand of position accuracy in Hi-tech scientific and industrial fields such as semiconductor industries, biomedical engineering, life science, nanotechnology, metrology/high precision measurement, agricultural, ground transportation and aerospace equipments etc. as position accuracy is a key performance objective in these fields. During the last three decades, the accuracy requirement in high-precision manufacturing and measuring processes has turned into from micrometer range to submicron and even nanometer range ${ }^{(1)}$. Therefore the vibration isolation is being become an important research topic in various precision measuring and micro manufacturing industries.

In practical circumstances, there are mainly two categories of disturbances take place into the vibration free platform and their effects should have to be attenuated. One is ground 
vibration transmitted to the isolation table through suspension and other is direct disturbance which takes place due to changing of weight and motion of equipments on the isolation table. The system with low stiffness isolator is better to attenuate the ground vibration as dynamic coupling between the vibration source and the vibration isolation table is weakened whereas high stiffness isolator is suitable to isolate the direct disturbance. Therefore zero stiffness and infinite stiffness are respectively ideal for reducing ground vibration and direct disturbance ${ }^{(2)}$. For ensuring quite vibration isolation, it needs infinite stiffness between the isolation table and base and low stiffness suspension between middle mass and base in the vibration isolation system. But realization of these two effects simultaneously by passive system is quite difficult as a trade-off is inevitable in conventional passive-type vibration isolation systems as well as their performances are exclusively limited.

In contrast, active-type vibration isolation systems are not, in principle, subjected to such performance limitation ${ }^{(2-3)}$. Recently, active vibration isolation systems are becoming popular for suppressing both the direct disturbance and the ground vibration simultaneously. Several researches are already conducted regarding active vibration isolation systems. Absolute-velocity feedback control system and state feedback control system are applied for vibration isolation ${ }^{(4-5)}$. However, most active systems are equipped with high performance sensor, such as servo-type accelerometer, which makes the system rather costlier than the passive-type system, roughly speaking, nearly ten times as expensive as passive ones.

To achieve the two necessary conditions of complete vibration isolation, the infinite stiffness and the low stiffness suspension in the control system simultaneously we proposed series combination of isolators and addition of middle mass between series connected isolators ${ }^{(6-7)}$. For active negative stiffness we have proposed zero power magnetic suspension controlled system with relative displacement sensors instead of servo-type accelerometers ${ }^{(8-9)}$. Zero power magnetic suspension control system has itself unique characteristic that it behaves as if it has negative stiffness, when an external force is applied to the suspended object. Therefore infinite stiffness can be realized by connecting a positive stiffness spring with magnetic suspension in series. We found that the negative stiffness controller with linear actuator shows the similar behavior as like zero power magnetic

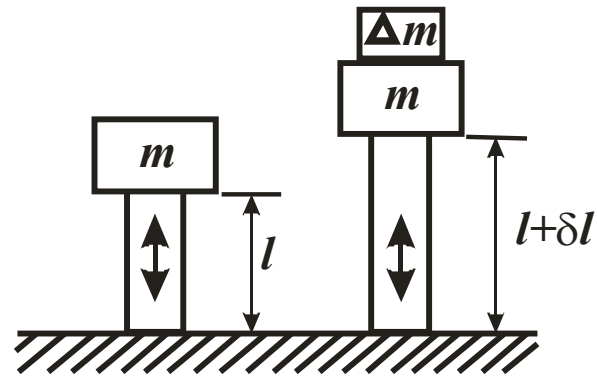

Fig. 1 Concept of negative stiffness

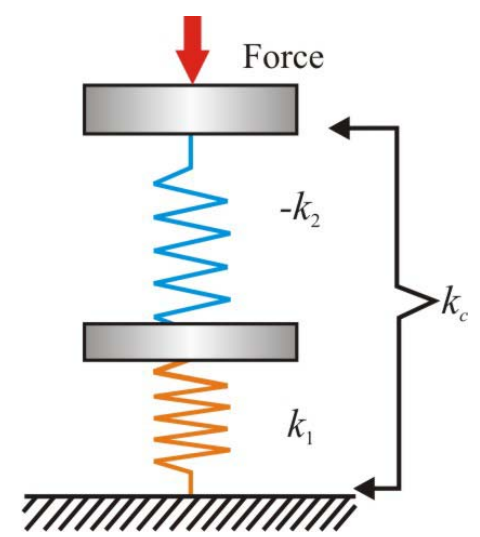

Fig. 2 Springs in series connection 
suspension control. The control technique, which is effective to widen the operation range of zero-power controllers, can be applied to the controller of negative stiffness as well ${ }^{(10)}$.

In this study, a three-axis freedom active vibration isolation system is developed using the concept of series combination of isolators. The displacement cancellation control strategy is applied to the developed system for horizontal vibration isolation associated with two horizontal translation motions and one rotational motion. In the control system the isolation table is connected with the middle mass through I-PD control instead of zero power magnetic suspension and middle mass is suspended with the base through PD control instead of conventional spring to make the system flexible. The corresponding controllers are arranged according to the mode base control regarding of three-degree-of freedom of motion control. The controller parameters are designed individually for every single axis without considering the effects of the motion of other axis and applied to the integrated whole system.

\section{Concept of displacement cancellation}

Infinite stiffness against direct disturbance and low stiffness suspension respect to base are two necessary conditions to suppress both disturbances. The principle of realizing infinite stiffness and respective zero compliance ${ }^{(8)}$ are explained in this section. The zero compliance means zero relative displacement of the isolation table with respect to the base.

In the aspect of negative stiffness, if there is a negative displacement - $\delta l$ (opposite direction of the force) due to adding of mass by $\Delta m$ then static negative stiffness of the mass can be presented as

$$
\frac{(\Delta m g)}{-\delta l}=k_{n},
$$

where the initial steady-state distance between base and mass is considered $l$ in Fig. 1. According to the principle of stiffness, when two isolators having stiffness coefficient $k_{1}$ and $k_{2}$ are connected in series as shown in Fig. 2, then the combine stiffness $k_{c}$ is given by

$$
k_{c}=\frac{k_{1} k_{2}}{k_{1}+k_{2}} .
$$

It shows that the combine stiffness of two isolators in series connection is lower than that of each isolator. However, if one isolator has negative stiffness and satisfy the following condition

$$
k_{1}=-k_{2} \text {, }
$$

then the combine stiffness $k_{c}$ of the corresponding isolators becomes infinite as follows,

$$
k_{c}=\frac{\left(-k_{2}\right) k_{2}}{-k_{2}+k_{2}}=\infty \text {. }
$$

Therefore, even if direct disturbance acts on the isolation table, the table has no steady-state displacement whatever low absolute value of the stiffness of each spring it is. In actual circumstance, however, it is difficult to maintain the condition $\left|k_{1}\right|=\left|k_{2}\right|$ in an integrated system while magnitude of applied load is being changed. To overcome this problem, the displacement cancellation control technique ${ }^{(11)}$ is applied in this paper.

In displacement cancellation control, it is not concerned about the stiffness of the isolators but relative position of the tables and corresponding actuator thrust force. The developed active system with the concept of the displacement cancellation is shown in Fig. 3. In steady-state, the displacement cancellation control technique provides rigid isolation table respect to middle mass and cancellation of the displacement of the isolation table respect to base caused due to the movement the middle mass. The position of the isolation table with respect to the base is determined by

$$
y=y_{1}+y_{2},
$$

where

$y_{1}$ : distance between middle mass and base. 
$y_{2}$ : distance between isolation table and middle mass.

The system with displacement cancellation control, actuators are guided in such way so that the expansion between the isolation table and the middle mass and contraction between the middle mass and the base are equal in magnitude $\left(\delta y_{1}=\delta y_{2}\right)$. Consequently, the position of the isolation table is to be kept in its original position as represented in below

$$
y^{\prime}=\left(y_{1}-\delta y_{1}\right)+\left(y_{2}+\delta y_{2}\right)=y,
$$

where $y^{\prime}$ and $y$ are distance of the isolation table from base regarding with and without force on the isolation table, respectively, $\delta y$ : displacement variation of the table.

\section{Control system design}

\subsection{Basic model of control system of single axis}

The displacement cancellation control can be achieved by using any linear actuator ${ }^{(11)}$. In this work, voice coil motors are used as actuators. To design the controller parameters for a specific axis motion control, the table is assumed to be guided by the actuator without any effect of motion of other axes. A basic single degree-of-freedom model with VCM for designing the controller is shown in Fig. 4. Here, the moving table with mass $m$ is assumed to be moved along $x$-axis of horizontal translation motions. The motion equation of the table guided by VCM is

$$
m \ddot{x}=f_{a}+f_{d},
$$

where

$m$ : mass of the table,

$x$ : displacement from original position,

$f_{a}$ : actuator's thrust force,

$f_{d}$ : direct disturbance acting on the table.

Thrust exerted by an actuator is proportional to coil current $i$, so the force $f_{a}$ can be expressed as

$$
f_{a}=k_{i} i
$$

where

$k_{i}$ : actuator's thrust force coefficient.

Permanent magnets of the VCM cause the magnetic fluxes which run in the magnetic circuit, and magnetic fields can be generated by moving charges (current), alternating current fields and permanent magnets. Assuming that leakage and fringing effects are neglected, the magnetic flux, $\Phi$ is confined to the physical structure. For the computation of the magnetic flux density, $B$, it is assumed that flux, $\Phi$ runs entirely within the magnetic

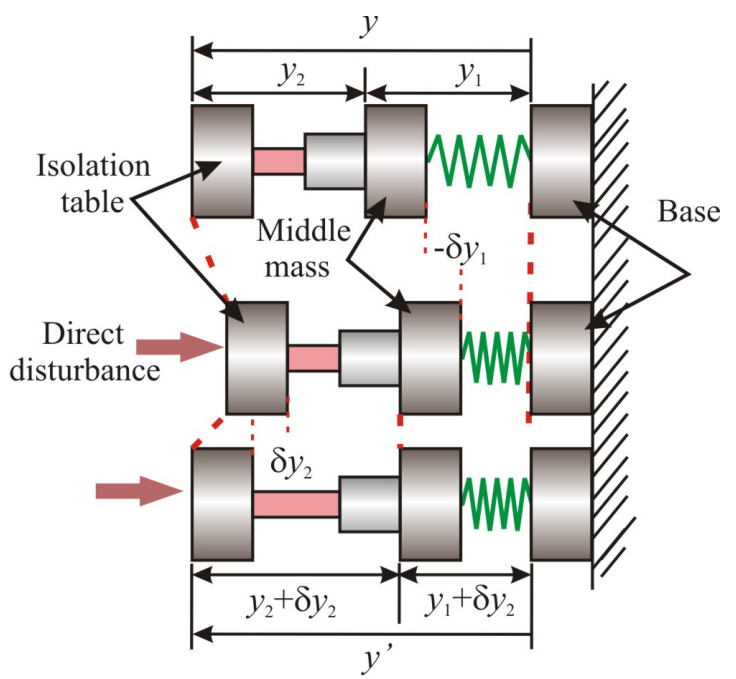

Fig. 3 Concept of displacement cancellation technique 
loop with constant coil winding section A, hence flux density could be measured as

$$
\phi=B A, \Rightarrow B=\frac{\phi}{A} .
$$

Therefore in case of VCM, thrust generated can be determined by

$$
F=B i L=k_{i} i,
$$

where

$B$ : magnetic flux density,

$i$ : coil current,

$L$ : length of the coil in the magnetic field

The values of $B$ and $L$ are constant as well as $k_{i}$ is constant for a particular VCM. Therefore it is understood that force generated by $\mathrm{VCM}$ is proportional to $i$. But in the research we measured VCM coefficient by measuring the force magnitude for per unit of current supply.

From Eqs. (7) and (8), the transfer function representation of the actuator's dynamics is given by

$$
X(s)=\frac{1}{s^{2}}\left(b_{0} I(s)+d_{0} F_{d}(s)\right),
$$

where each Laplace-transformed variable is denoted by its capital and

$$
b_{0}=\frac{k_{i}}{m}, \quad d_{0}=\frac{1}{m} .
$$

If a constant frictional coefficient $\mu$ is considered at the contact points of the table and vertical supports, then the transfer function representation of the actuator's dynamics becomes as follows

$$
X(s)=\frac{1}{s^{2}+a_{1} s}\left(b_{0} I(s)+d_{0} F_{d}(s)\right),
$$

where

$$
a_{1}=\mu g \text {, and } \mu \text { : frictional coefficient. }
$$

\subsection{Basic model of control system of three axes}

Figure 5 shows a schematic diagram of a developed three-degree-of-freedom vibration isolation system. The system consists of two square shape moving tables (isolation table, middle mass) are driven by voice coil motors (VCMs) located at the vertices of an equilateral square. Each table is supported vertically respect to the base through ball bearing consequently it can move about horizontal plane associated with two horizontal translation axes and vertical rotation axis rotation $(x, y$ and $\theta z)$ shown in Fig. 6. Therefore three-degree-of-vibration isolation can be achieved by the developed system. Since both tables are supported vertically from base individually, there induced friction is not associated with the relative velocities of the tables. Hence the velocities of the tables respect to base are the variable parameters to the frictional damping coefficient shown in Eq. (13). In the developed system, the isolation table is guided by the actuators mounted on the middle table whereas the middle table is driven by the actuators located on the base.

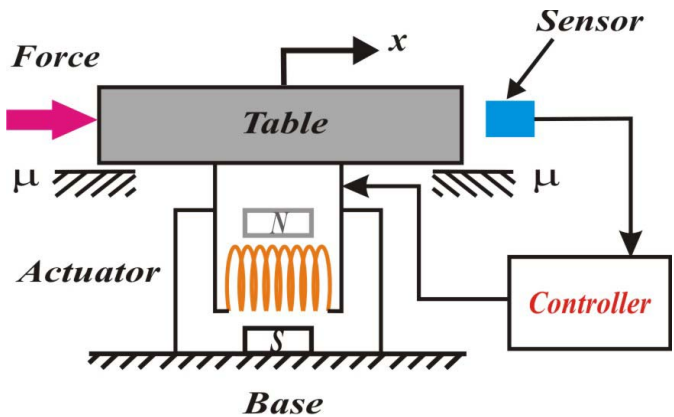

Fig. 4 Basic model of horizontal suspension with VCM 
Therefore the relative movements regarding isolation table to middle table and middle table to base are controlled in this experiment. It is assumed that rotational displacement $\theta_{\mathrm{z}}$ is so small, hence, $\cos \theta_{\mathrm{z}}=1$ and $\sin \theta_{\mathrm{z}}=\theta_{\mathrm{z}}$. The three degree motion equations of the isolation table can be written as

$$
R_{m}\left[\begin{array}{c}
\ddot{x} \\
\ddot{y} \\
\ddot{\theta}_{z}
\end{array}\right]=-R_{f}\left[\begin{array}{c}
\dot{x} \\
\dot{y} \\
\dot{\theta}_{z}
\end{array}\right]+\left[\begin{array}{c}
f_{x}{ }^{a} \\
f_{y}{ }^{a} \\
T_{z}{ }^{a}
\end{array}\right]+\left[\begin{array}{l}
f_{x}{ }^{d} \\
f_{y}^{d} \\
T_{z}{ }^{d}
\end{array}\right],
$$

where

$R_{m}$ : mass matrix,

$R_{f}$ : frictional damping effect matrix,

$x, y$ : linear displacement along $x$ and $y$-axis,

$\theta_{\mathrm{z}}$ : angular displacement about $z$-axis,

$f_{x}^{a}, f_{y}^{a}, T_{z}^{a}:$ forces and torque generated by the actuators,

$f_{x}^{d}, f_{y}^{d}, T_{z}^{d}$ : disturbing forces and torque acting on the table.

$$
R_{m}=\left[\begin{array}{ccc}
m & 0 & 0 \\
0 & m & 0 \\
0 & 0 & I_{z}
\end{array}\right], \quad R_{f}=\left[\begin{array}{ccc}
\mu m g & 0 & 0 \\
0 & \mu m g & 0 \\
0 & 0 & u m g R^{2}
\end{array}\right],
$$

$m$ : mass of the table,

$I_{z}$ : inertia momentum of the table about $z$-axis,

$\mu$ : frictional coefficient,

$R$ : distance between centre point and vertical supporting point of the table.

The thrust forces and the resisting torque are generated by actuators presented in below

$$
f_{x}^{a}=k_{i x} i_{x}, f_{y}^{a}=k_{i y} i_{y}, T_{z}^{a}=k_{i z} i_{z} l,
$$

where

$k_{i x}, k_{i y}, k_{i z}:$ actuator thrust coefficient along corresponding axis,

$i_{x}, i_{y}, i_{z}$ : control current along the corresponding axis,

$2 l$ : distance between two actuators in opposite vertex of the equilateral square.

Hence the transfer function representation of the actuator's dynamics for 3-DOF motion of the system can be described as follows

$$
\left[\begin{array}{c}
X(s) \\
Y(s) \\
\theta(s)
\end{array}\right]=\left[\begin{array}{c}
\frac{1}{m s^{2}+\mu m g s} \\
\frac{1}{m s^{2}+\mu m g s} \\
\frac{1}{I_{z} s^{2}+\mu m g R^{2} s}
\end{array}\right]\left(\begin{array}{l}
k_{i x} I_{x}(s)+f_{x}{ }^{d} \\
k_{i y} I_{y}(s)+f_{y}^{d} \\
k_{i z} I_{z}(s) l+T_{z}{ }^{d}
\end{array}\right) .
$$

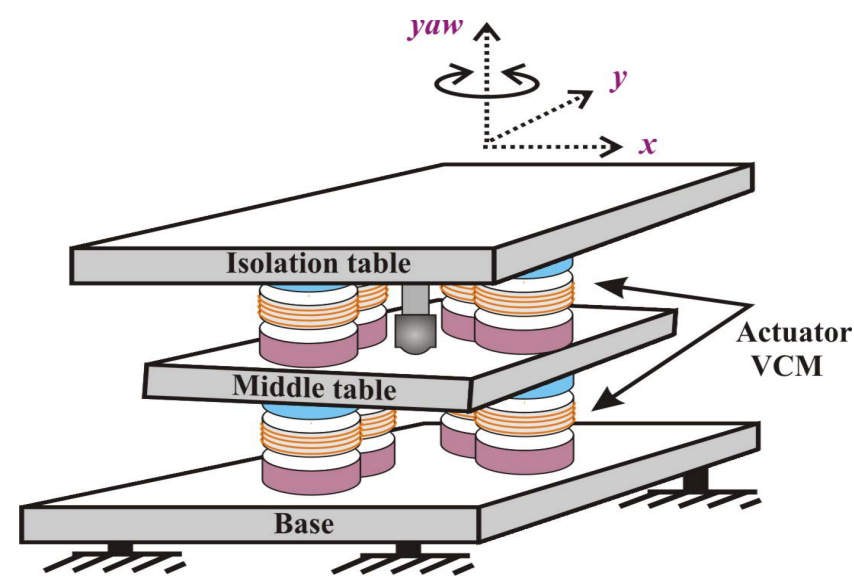

Fig. 5 Schematic diagram of the 3-DOF vibration Isolation system 
The three modes controlled system is realized by designing the controller of each single mode individually and applied to the system simultaneously. In the following section, the procedure of designing the controller for a single axis ( $x$-axis) is described.

\subsection{Design of controllers of displacement cancellation technique}

In displacement cancellation control technique, the actuators are guided in such way so that they can create displacement of the isolation table in the opposite direction of the middle table in order to cancel the effect of the displacement of the middle table on the isolation table. For achieving this effect, the isolation table is directed by PD control respect to the middle table and is directed by integral control respect to the base. The middle table is controlled by PD (positive stiffness) control respect to base. The applied force on the isolation table goes to the middle table as well as it moves along the direction of the force due to PD control. Consequently the isolation table also moves along the same direction which is cancelled by subtracting the displacement of middle table from the integral input of the I-PD control in the isolation table as shown in Fig.7. There is no relative displacement of the isolation table respect to the base due to I-PD control in the isolation table. Moreover the vibration isolation systems must have the capability to attenuate the ground vibration transmitted from floor to the isolation table. An effective filter between the middle table and the isolation table with low stiffness PD control can suppress the transmitting of ground vibration competently ${ }^{(12)}$. Therefore an electric filter (low-pass filter) is considered between isolation table and the middle table (mass) in mode control network of our developed system.

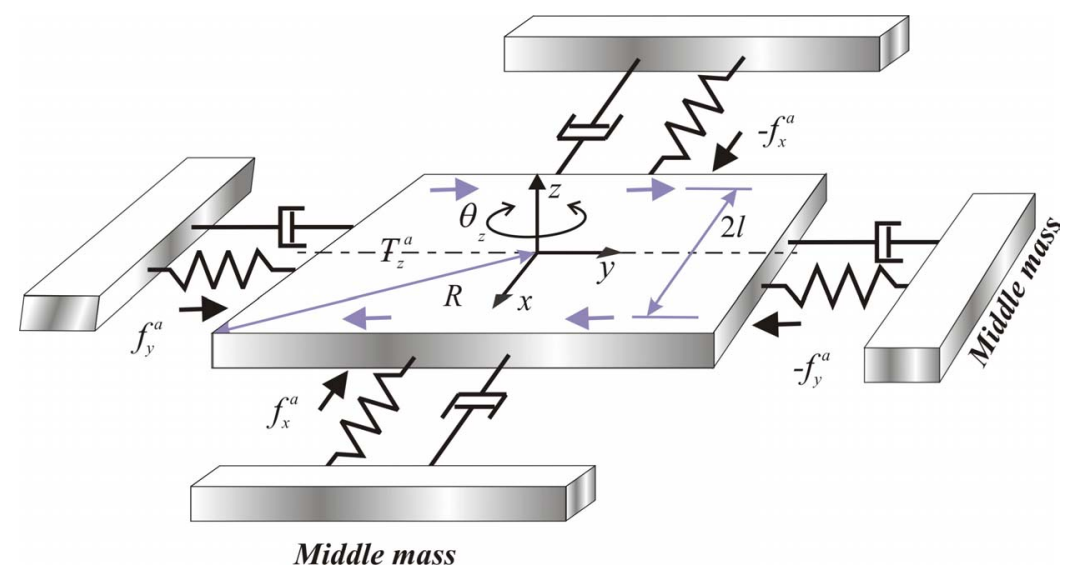

(a) Isolation table

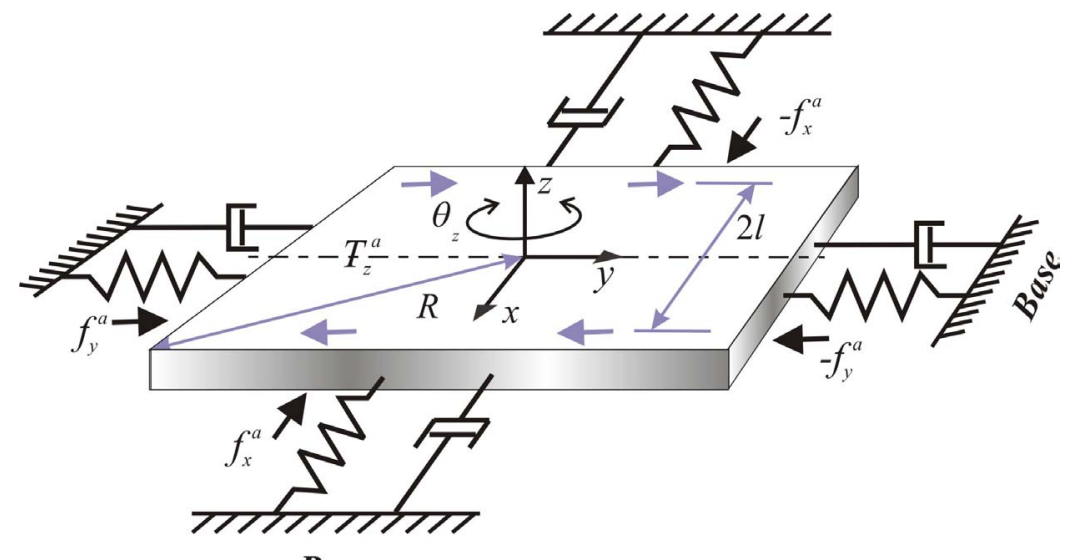

Base

(b) Middle table

Fig. 6 Coordinate system of 3-DOF system 


\subsubsection{Design of displacement cancellation controller}

The respective controller for every single axis motion of each table is designed separately without considering the effect of internal interference of other table and axes. The corresponding controller parameters are determined according to the pole assignment method with low-pass filter of unique effect. The procedure of designing the controller along $x$-axis is presented in this paper. The block diagram of basic structure of the I-PD controller is shown in Fig. 8 and corresponding control current of the I-PD control can be written as follows

$$
i(t)=-P_{i} \int x d t-P_{d} x-P_{v} \dot{x} .
$$

The Laplace-transformation of the Eq. (15) is as follows

$$
I(s)=-\frac{P_{i}}{s} X(s)-P_{d} X(s)-P_{v} s X(s) .
$$

Substituting of Eq. (16) into Eq. (12) leads to

$$
\frac{X(s)}{F_{d}(s)}=\frac{d_{0} s}{\hat{t}_{c}(s)},
$$

where characteristics equation of the I-PD control system is expressed by

$$
\hat{t}_{c}(s)=s^{3}+\left(a_{1}+b_{0} P_{v}\right) s^{2}+b_{0} P_{d} s+b_{0} P_{i} .
$$

To obtain the system like as the ideal system of 3 rd-order characteristic equation formed as

$$
\hat{t}_{d}(s)=\left(s^{2}+2 \hat{\zeta}_{1} \hat{\omega}_{1} s+\hat{\omega}_{1}^{2}\right)\left(s+\hat{\omega}_{2}\right)=\left(s^{3}+\hat{\alpha}_{2} s^{2}+\hat{\alpha}_{1} s+\hat{\alpha}_{0}\right),
$$

it needs to match the coefficients of the Eqs. (18) and (19). Finally the controller parameters are achieved as follows

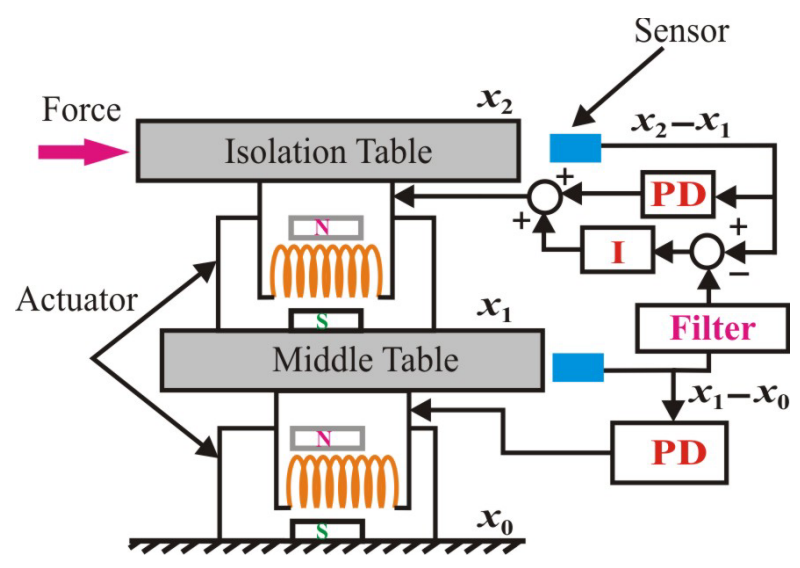

Fig. 7 Schematic diagram of displacement cancellation control technique

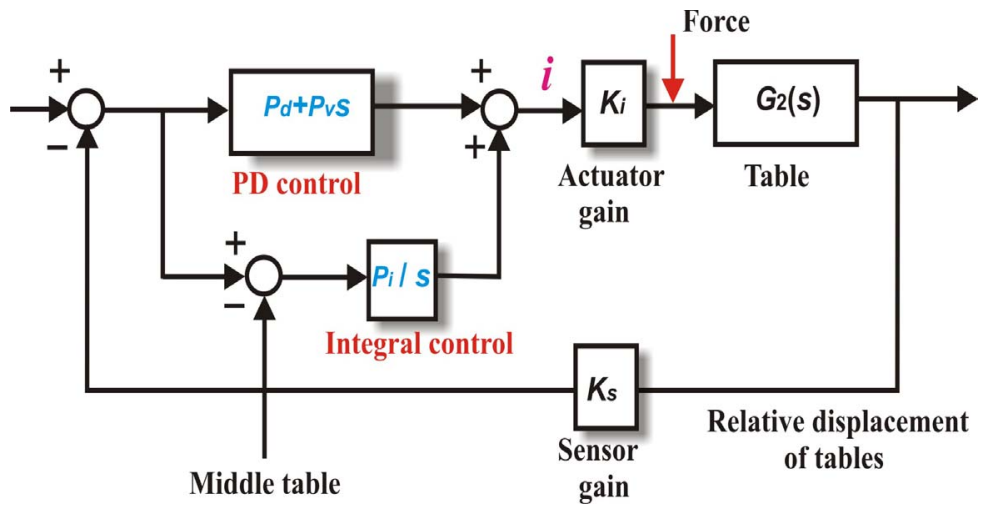

Fig. 8 Block diagram of I-PD control system 


$$
P_{i}=\frac{\hat{\alpha}_{0}}{b_{0}}, \quad P_{d}=\frac{\hat{\alpha}_{1}}{b_{0}}, \quad P_{v}=\frac{\hat{\alpha}_{2}-a_{1}}{b_{0}},
$$

where

$$
\hat{\alpha}_{2}=2 \hat{\zeta}_{1} \hat{\omega}_{1}+\hat{\omega}_{2}, \hat{\alpha}_{1}=2 \hat{\zeta}_{1} \hat{\omega}_{1} \hat{\omega}_{2}+\hat{\omega}_{1}^{2}, \quad \hat{\alpha}_{0}=\hat{\omega}_{1}^{2} \hat{\omega}_{2} .
$$

Here symbols are defined as follows. $\hat{\zeta}$ : damping ratio in designing the I-PD controller, $\hat{\omega}$ : angular velocity in designing the I-PD controller. These two parameters define the set of poles of the ideal control system.

To make the system convenient, the middle table is also guided by active isolators (VCMs) instead of normal spring. To accomplish the system with soft suspension, actuator of the middle table is driven through PD control with low stiffness.

To design the PD controller for a particular positive stiffness to the middle table, the basic motion equation (12) is followed and all parameters are considered according to the middle table. The basic structure of the PD controller is shown in Fig. 9. The characteristics equation of the PD control system can be expressed by

$$
t_{c}^{\prime}(s)=s^{2}+\left(a_{1}+b_{0} P_{v}\right) s+b_{0} P_{d} .
$$

The parameters of the PD control for a particular positive stiffness $\left(k_{\mathrm{p}}\right)$ are determined by the following manner. The direct disturbance is assumed to be stepwise so that it can be modeled as

$$
F_{d}(s)=\frac{F_{0}}{s} \quad\left(F_{0}: \text { const }\right) .
$$

It is assumed that the controller is selected to stabilize the closed loop system. The steady-state displacement $x(\infty)$ is given by

$$
\frac{x(\infty)}{F_{0}}=\lim _{s \rightarrow 0} s \frac{d_{0}}{s^{2}+\left(a_{1}+b_{0} P_{v}\right) s+b_{0} P_{d}} \cdot \frac{1}{s}=\frac{d_{0}}{b_{0} P_{d}} .
$$

If the control system provides positive stiffness of magnitude $k_{p}$, then the following equation must be satisfied

$$
\frac{d_{0}}{b_{0} P_{d}}=\frac{1}{k_{p}} \text {. }
$$

To obtain the system like as the effect of an ideal system of 2nd-order characteristic equation formed as below

$$
t_{d}^{\prime}(s)=\left(s^{2}+2 \zeta_{1}^{\prime} \omega_{1}^{\prime} s+\omega_{1}^{\prime 2}\right)=\left(s^{2}+\alpha_{1}^{\prime} s+\alpha_{0}^{\prime}\right),
$$

we could do with to the match coefficients of the Eqs. (20) and (24) and by the Eq (23), finally the controller parameters are determined as follows

$$
P_{d}=\frac{k_{n} d_{0}}{b_{0}}, \quad P_{v}=\frac{\alpha_{1}^{\prime}-a_{1}}{b_{0}}
$$

where

$$
\alpha_{1}^{\prime}=2 \zeta_{1}^{\prime} \omega_{1}^{\prime}, \quad \alpha_{0}^{\prime}=\omega_{1}^{\prime 2}
$$

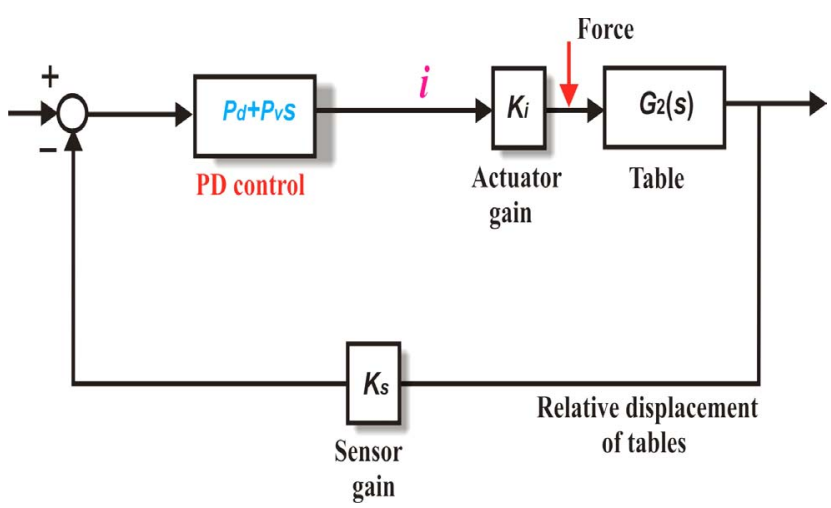

Fig. 9 Block diagram of PD control system 
Here symbols are defines as follows. $\zeta^{\prime}$ : damping ratio in designing the PD controller, $\omega^{\prime}$ : angular velocity designing the PD controller and these two parameters define the set of poles of the control system.

\section{Theoretical analysis}

\subsection{Basic equation}

The middle table is modeled by positive stiffness $\left(k_{p}\right)$ controller through PD control and the isolation table respect to the middle table is modeled by I-PD controller with low-pass filter in between them. To find the motion equations of the tables of the integrated system, the basic motion equation of the isolation table and that of the middle table along $x$ axis (translation motion) are exploited here. In the corresponding equations of the integrated system, suffix 1 and 2 with the respective symbols represent the parameters regarding the middle table and the isolation table, respectively. Motion equations of the tables of integrated system along horizontal translation (x-axis) are written in below.

$$
\begin{aligned}
& m_{1} \ddot{x}_{1}+c_{1}\left(\dot{x}_{1}-\dot{x}_{0}\right)=k_{i 1} i_{1}-k_{i 2} i_{2}, \\
& m_{2} \ddot{x}_{2}+c_{2}\left(\dot{x}_{2}-\dot{x}_{1}\right)=k_{i 2} i_{2}+f_{d},
\end{aligned}
$$

where

$x_{0}, x_{1} x_{2}$ : displacement of the floor, middle table and isolation table, respectively,

$c$ : friction viscous coefficient $=\mu m g$.

Meanings of the other symbols in this section are same as mentioned in the section of basic model (section 3). The Eqs. (25) and (26) respectively can be written in Laplace transformation form given in below

$$
\begin{aligned}
& m_{1} s^{2} X_{1}(s)+c_{1} s\left(X_{1}(s)-X_{0}(s)\right)=k_{i 1} I_{1}(s)-k_{i 2} I_{2}(s), \\
& m_{2} s^{2} X_{2}(s)+c_{2} s\left(X_{2}(s)-X_{1}(s)\right)=k_{i 2} I_{2}(s)+F_{d}(s) .
\end{aligned}
$$

The control current for positive stiffness (PD) control to the middle table and I-PD control to the isolation table with the effect of low-pass filter, respectively are determined by

$$
\begin{aligned}
& I_{1}(s)=-\left(P_{d 1}+P_{v 1} s\right)\left(X_{1}(s)-X_{0}(s)\right), \\
& I_{2}(s)=-\frac{P_{i}}{s}\left\{G_{F}(s)\left(X_{1}(s)-X_{0}(s)\right)+\left(X_{2}(s)-X_{1}(s)\right)\right\} \\
& -\left(P_{d 2}+P_{v 2} s\right)\left(X_{2}(s)-X_{1}(s)\right),
\end{aligned}
$$

where $G_{F}(s)$ is the transfer function of the low-pass filter that satisfies the condition

$$
G_{F}(0)=1 \text {. }
$$

Solving of the Eqs. (27) and (28) and considering the Eqs. (29) and (30), leads to integrated transfer function of the whole close loop control system given as follows

$$
\begin{aligned}
X_{1}(s)= & {\left[\frac{\left(c_{1} s+t_{1}(s)-t_{3}(s) G_{F}(s)\right)\left(m_{2} s^{2}+c_{2} s+t_{2}(s)+t_{3}(s)\right)+t_{3}(s) G_{F}(s)\left(t_{2}(s)+t_{3}(s)\right)}{t_{t c}(s)}\right] } \\
X_{0}(s)+\frac{\left(t_{2}(s)+t_{3}(s)\right)}{t_{t c}(s)} F_{d}(s) & \\
X_{2}(s)= & {\left[\frac{t_{3}(s) G_{F}(s)\left(m_{1} s^{2}+c_{1} s+t_{1}(s)+t_{2}(s)+t_{3}(s)\left(1-G_{F}(s)\right)\right)}{t_{t c}(s)}\right] } \\
& \left.+\frac{\left(c_{1} s+t_{1}(s)-t_{3}(s) G_{F}(s)\right)\left(c_{2} s+t_{2}(s)+t_{3}(s)\left(1-G_{F}(s)\right)\right)}{t_{t c}(s)}\right] X_{0}(s) \\
& +\left[\frac{\left(m_{1} s^{2}+c_{1} s+t_{1}(s)+t_{2}(s)+t_{3}(s)\left(1-G_{F}(s)\right)\right)}{t_{t c}(s)}\right] F_{d}(s)
\end{aligned}
$$

where

$$
\begin{aligned}
& t_{1}(s)=k_{i 1}\left(P_{d 1}+P_{v 1} s\right), \\
& t_{2}(s)=k_{i 2}\left(P_{d 2}+P_{v 2} s\right),
\end{aligned}
$$




$$
\begin{gathered}
t_{3}(s)=k_{i 2}\left(\frac{P_{i}}{s}\right) \text { and } \\
t_{t c}(s)=\left\{\left(m_{1} s^{2}+c_{1} s+t_{1}(s)+t_{2}(s)+t_{3}(s)\left(1-G_{F}(s)\right)\right)\left(m_{2} s^{2}+c_{2} s+t_{2}(s)+t_{3}(s)\right)\right. \\
\left.\quad-\left(t_{2}(s)+t_{3}(s)\right)\left(c_{2} s+t_{2}(s)\right)+t_{3}(s)\left(1-G_{F}(s)\right)\right\} .
\end{gathered}
$$

Since it is assumed that the controller is selected to stabilize the close loop system. Thus it is to be noted that $t_{1}(s), t_{2}(s)$ and $t_{t c}(s)$ must satisfy the condition (23), and the corresponding condition for positive stiffness is given as follows,

$$
\frac{d_{0}}{b_{0} P_{d}}=\frac{1}{k_{i 1} P_{d 1}}=\frac{1}{k_{p}} .
$$

\subsection{Response to direct disturbance}

To estimate the stiffness to direct disturbance, the direct disturbance $f_{d}$ is assumed to be stepwise, that is

$$
F_{d}=\frac{F_{0}}{s}\left(F_{0}: \text { const }\right) .
$$

When there is no ground vibration, the steady-state displacement of the isolation table against to direct disturbance is obtained as

$$
\frac{x_{2}(\infty)}{F_{0}}=\lim _{s \rightarrow 0} s \frac{\left(m_{1} s^{2}+c_{1} s+t_{1}(s)+t_{2}(s)+t_{3}(s)\left(1-G_{F}(s)\right)\right)}{t_{t c}(s)} \cdot \frac{1}{s}=\frac{k_{i 1} P_{d 1}+k_{i 2} P_{d 2}}{\frac{1}{s}\left(k_{i 2} P_{i} k_{i 1} P_{d 1}\right)}=0 .
$$

The equation (35) shows that the control system with corresponding controller has infinite stiffness or zero compliance statically between isolation table and base because there is no steady-state deflection even in the presence of stepwise disturbances acting on the table.

On the other hand, the steady-state displacement of the middle table and relative displacement between two tables, respectively are obtained as follows

$$
\begin{aligned}
& \frac{x_{1}(\infty)}{F_{0}}=\lim _{s \rightarrow 0} s \frac{\left(t_{2}(s)+t_{3}(s)\right)}{t_{t c}(s)} \cdot \frac{1}{s}=\frac{\frac{1}{s}\left(k_{i 2} P_{i}\right)}{\frac{1}{s}\left(k_{i 2} P_{i} k_{i 1} P_{d 1}\right)}=\frac{1}{k_{i 1} P_{d 1}}=\frac{1}{k_{p}},(\because(34)) \\
& \frac{x_{2}(\infty)-x_{1}(\infty)}{F_{0}}=-\frac{1}{k_{p}} .
\end{aligned}
$$

The equations (36) and (37) indicate that the middle table moves to the same direction as the applied force and the relative displacement of the isolation table to the middle table is opposite of the direction of the applied force as there is a negative stiffness between the isolation table and the middle table. Mizuno et $a l^{(7)}$ found the same behavior in case of vertical plane vibration isolation.

\subsection{Control strategy}

In the developed active system, each horizontal translation motion is controlled by two actuators and rotational motion is controlled by combine effort of all four actuators those control the two translation motions simultaneously. Therefore, control network is arranged in mode control way so that less number of actuators (compared to control axes) can control the entire motion of freedom (two translations and one rotation) shown in Fig. 10. Every single actuator (VCM) in our system can generate force along to and fro direction (single axis). Here actuator 1 and 3 generate thrust and control the motion along Y-axis whereas actuator 2 and 4 can generate thrust force along $\mathrm{X}$-axis and control the respective motion. The rotational torque on the table along Yaw axis can be produced by actuator 1 and 3 when they generate equal force along the opposite direction of each other simultaneously. Similarly actuator 2 and 4 can generate torque on the table along Yaw axis. For clockwise 
angular displacement the actuators generate equal thrust along the directions as like shown in Fig.11 so that anti-clock wise resisting torque is produced about center point of the table. Therefore the system can provide rotational torque and translation thrust by adding the corresponding axis control single simultaneously without hampering the each other shown in Fig. 10.

In the case of mode control to the developed system, control current is designed for each mode $\left(x, x\right.$ and $\left.\theta_{z}\right)$ individually according to the displacement signal as shown in Fig. 10. The displacements of individual mode are measured by sensors and respective displacements are considered as the input signal of the controller to find the corresponding output control signal of the controller. Again the control currents $\left(i_{1}, i_{2}, i_{3}, i_{4}\right)$ for four VCMs are calculated from the outputs $\left(i_{x}, i_{y}, i_{\theta z}\right)$ of the respective controllers.

\section{Experiments}

\subsection{Experimental setup}

A photograph of the developed vibration isolation system is shown in Fig. 12. The system consists with two moving tables (isolation and middle table) and each moving table is supported by four vertical ball point supports of free ball bearing respect to base. Therefore moving table has freedom of motions along two horizontal translation motions and one rotational motion along vertical axis. In the developed system each moving table is controlled by four actuators (VCMs) and six eddy current gap sensors are used to detect its displacement shown in Fig. 10. The actuators which guide the isolation table are placed between two tables whereas the middle table driven actuators are installed between the base and the middle table shown in Fig. 5.13. Moreover two VCMs are mounted on the isolation table and they are fixed to the upper stage to generate the direct disturbance acting on the isolation table as well.

The isolation table is dimensioned by $620 \mathrm{~mm} \times 620 \mathrm{~mm}$ with thickness $10 \mathrm{~mm}$ and total mass of the isolation table is $20.2 \mathrm{~kg}$. Here, all dimensions of the isolation table are considered on the basis of standard driver seat dimensions of heavy vehicle with standard human weight as our active developed system would be proposed for seat suspension of

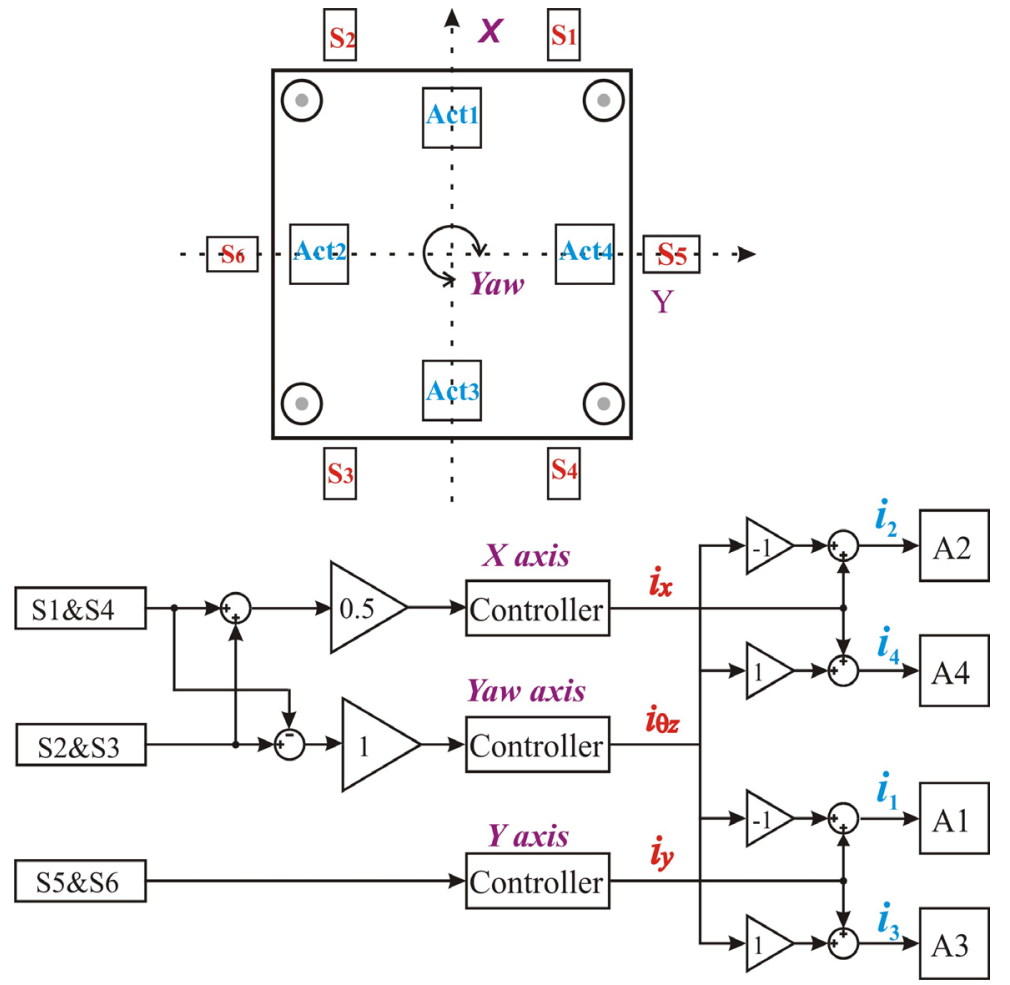

Fig. 10 Block diagram of the mode control system 


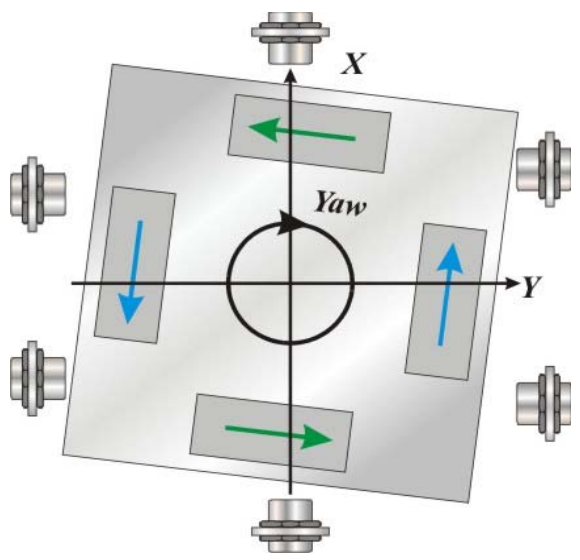

Fig. 11 Rotational displacement and actuator force direction

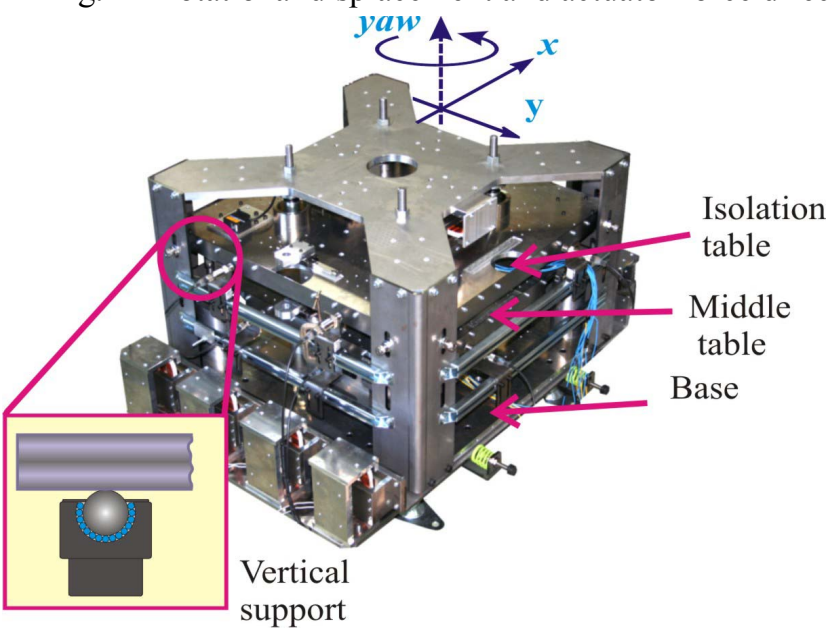

Fig. 12 Photograph of the developed vibration isolation system

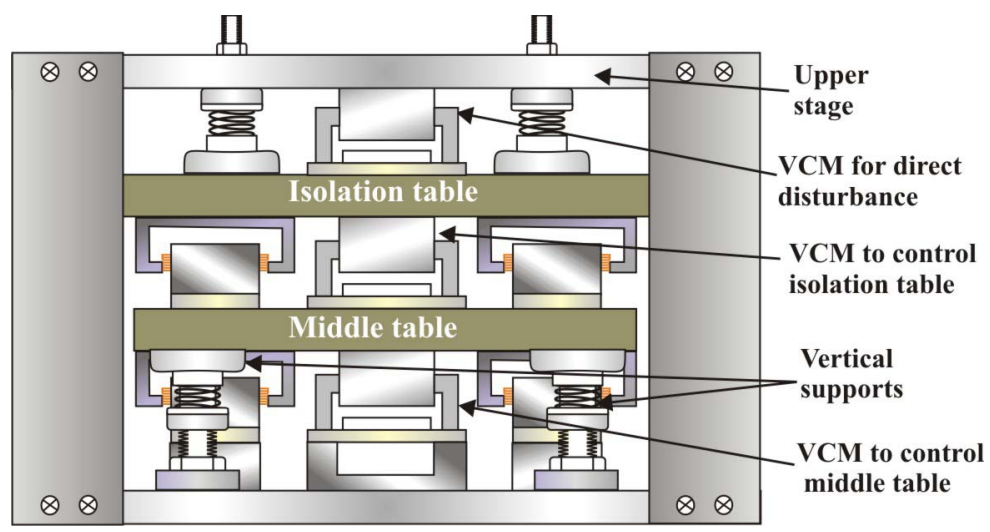

Fig. 13 Schematic diagram of the developed system

construction vehicle. On the other hand middle table is dimensioned by $530 \mathrm{~mm}$ x $530 \mathrm{~mm}$ with thickness $10 \mathrm{~mm}$ and the mass is $15.5 \mathrm{~kg}$ to support the required specification of the isolation table mentioned in above.

Linear displacement of the table is measured by taking average displacements of two end points of the table. Moreover the rotational displacement of the table is measured by taking difference of the displacements of two sensors in the same line of one side of the table. Designed control algorithms are implemented with a digital signal processor supplied by dSPACE ${ }^{T M}$. 


\subsection{Experimental results}

To check the performances of the displacement cancellation control system to direct disturbance, several experiments have been conducted where the effect of filter is neglected $\left(G_{F}(s)=1\right)$. For generating disturbances along translation axis, VCMs are placed so that the generated disturbances on the isolation table are along the center line of the table. On the other hands, the torque along rotational axis, the VCMs are placed in opposite direction so that the generated disturbance torque is about the centre point of the table. The static and the dynamic responses along the two horizontal translation motions ( $x$ and $y$ axis) are almost same for a particular system, thus in the discussion of the experimental results, system responses along one translation axis ( $x$-axis) and rotational axis (yaw-axis) are presented.

The figures 14(a) and 14(b) show the static characteristics of the control system using mode control of displacement cancellation technique along the horizontal translation axis ( $x$-axis) and rotational axis (yaw-axis), respectively. It is observed that the isolation table keeps almost the same position, while the position of the middle table is changed proportionally to the step wise static load and torque along translation axis and rotational axis, respectively. Here, experiments are conducted for same set of poles $(5 \mathrm{~Hz}$ of resonance frequency $\left.\omega_{x}=\omega_{z}=2 \pi \times 5, \zeta_{x}=\zeta_{z}=0.5\right)$ in designing the controllers in the both translational ( $x$-axis) and rotational direction (z-axis). In this experiment, it is perceived that the translation displacement of the middle table to direct static load is well linear compared to the rotational displacement to direct static torque.

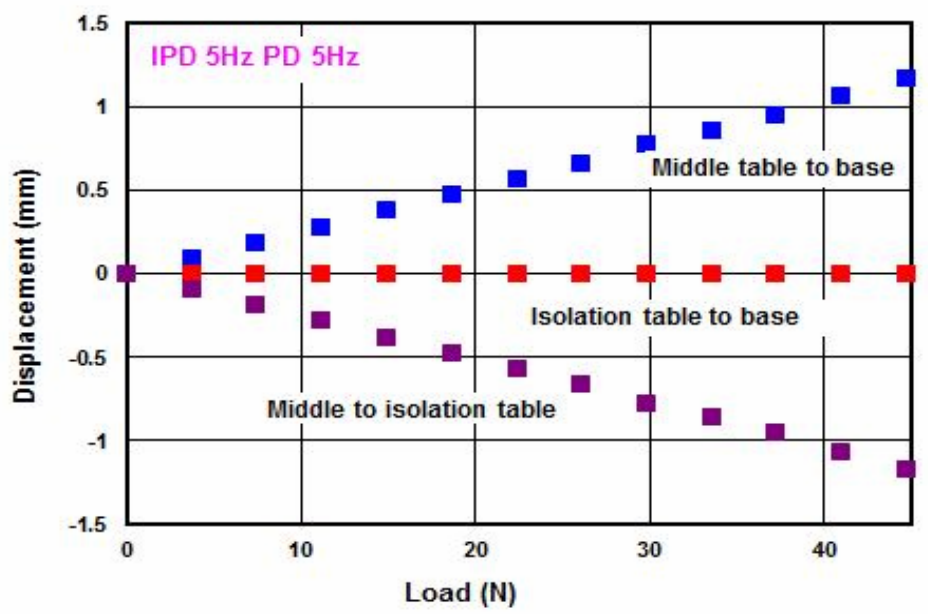

(a) horizontal translation ( $x$-axis)

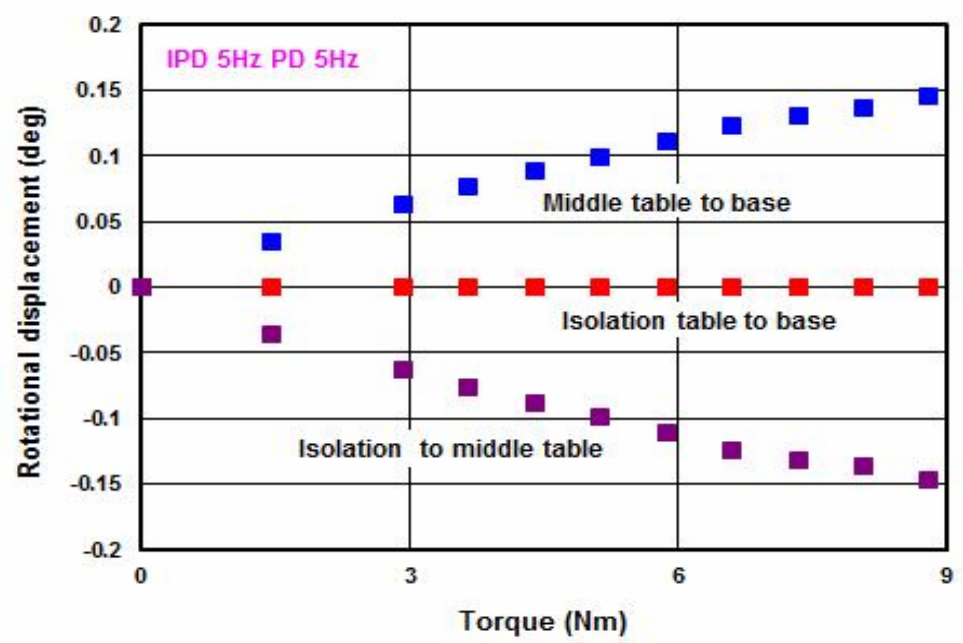

(b) horizontal rotation (yaw axis)

Fig. 14 Static response of the system to direct disturbance 
To find step responses of the control system, the step input to the corresponding controller is generated by applying a certain amount of load $(10 \times 2 \mathrm{~N})$ and torque $(10 \mathrm{~N} \times$ distance between two VCMs) suddenly about the center point of the isolation table. The behaviors of the step input to control system along the translation axis $(x)$ and rotational axis are illustrated in Figs. 15(a) and 15(b), respectively. The positions of the tables for a certain step input over the time period are drawn in this figure. Here it is observed that the isolation table returns to its original position after settling time whereas middle table maintains certain displacement in both directions of motion. The displaced position of the middle table depends upon the conditions of the applied controller. Time required (settling time) to become the system stable is same in both directions as experiments are conducted under the same set of poles in designing the controllers $\left(\omega_{x}=\omega_{z}=2 \pi \times 5, \zeta_{x}=\zeta_{z}=0.5\right)$.

In this analysis it was observed that there is transient displacement of the isolation table. The transient displacement of the isolation table depends on the dynamic behavior of the middle table (positive stiffness) to base and isolation table to middle table (negative stiffness). The dynamic behavior is defined by set of poles of the characteristic equation of the control system. If the poles are selected so that the dynamic behaviors of the positive and negative stiffness mentioned in above are same then transient displacement of the isolation table will not be appeared. But in actual practice it is difficult to turn into the same dynamic behavior as poles depend on several parameters (damping ratio, resonance frequency, actuator capacity, frictional effect etc.). Thus theoretically it is possible to omit

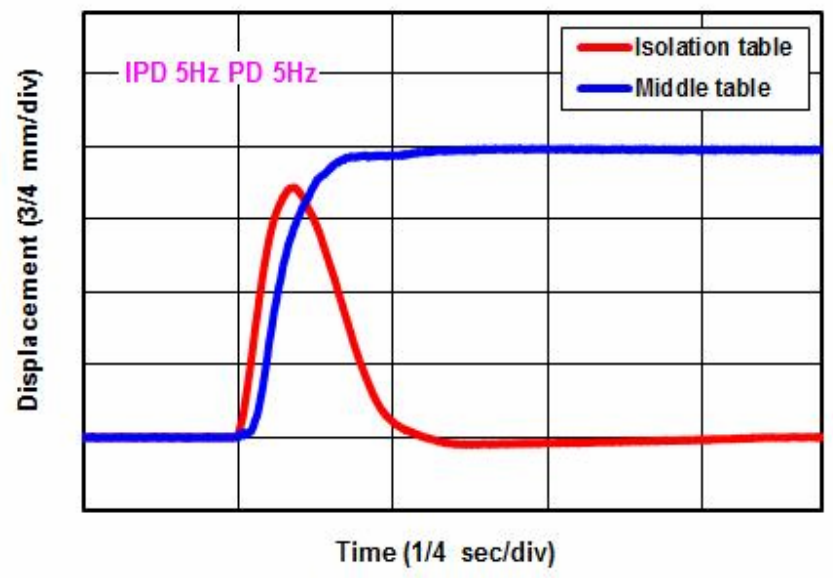

(a) Translation motion (x-axis)

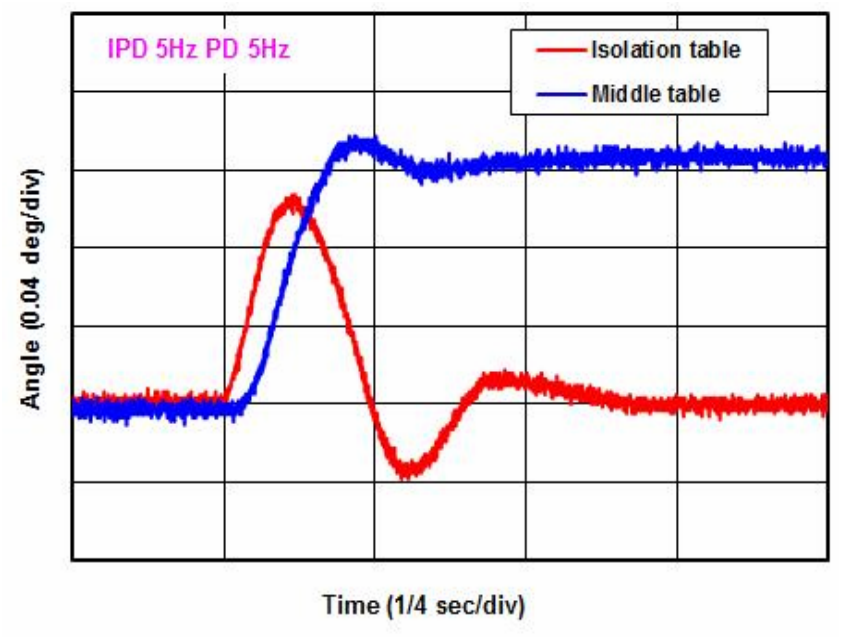

(b) Rotational motion (yaw-axis)

Fig. 15 Step responses of the system to step input (direct disturbance) 
the transient peak of the isolation table. Moreover the including of feed forward control to the main control stream can reduce the transient displacement respect to know disturbance. In the future work, it would be continued to minimize the transient peak.

However, there is a lager damping effect in translation movement compared to the rotational movement. This might be due to larger sliding contact area as well as larger frictional effect in translational movement.

The frequency responses of the control system to direct disturbance along horizontal translation ( $x$-axis) and horizontal rotation (yaw-axis) for different sets of poles in designing the controllers are represented in Figs. 16(a) and 16(b), respectively. For different sets of poles, the $\mathrm{dB}$ gain and phase are drawn over the frequency range in this figure. The sets of poles in designing the controller are defined by considering different resonances of the isolation table and the middle table with constant damping ratio. The experimental conditions as well as the sets of poles in designing the controllers are mentioned in legend of Fig. 16. For example, "IPD 5Hz PD 5Hz" indicates $\hat{\omega}_{1}=\hat{\omega}_{2}=\omega_{1}^{\prime}=2 \pi \times 5.0 \mathrm{rad} / \mathrm{sec}$ mentioned in Eqs. (19) and (24). From this figure it is confirmed that the dynamic disturbances could be suppressed very well by displacement cancellation control system as $\mathrm{dB}$ gain decreases sharply at higher frequency region and low $\mathrm{dB}$ gain is recorded at very low frequency range. The dynamics responses of the control system along rotational axis

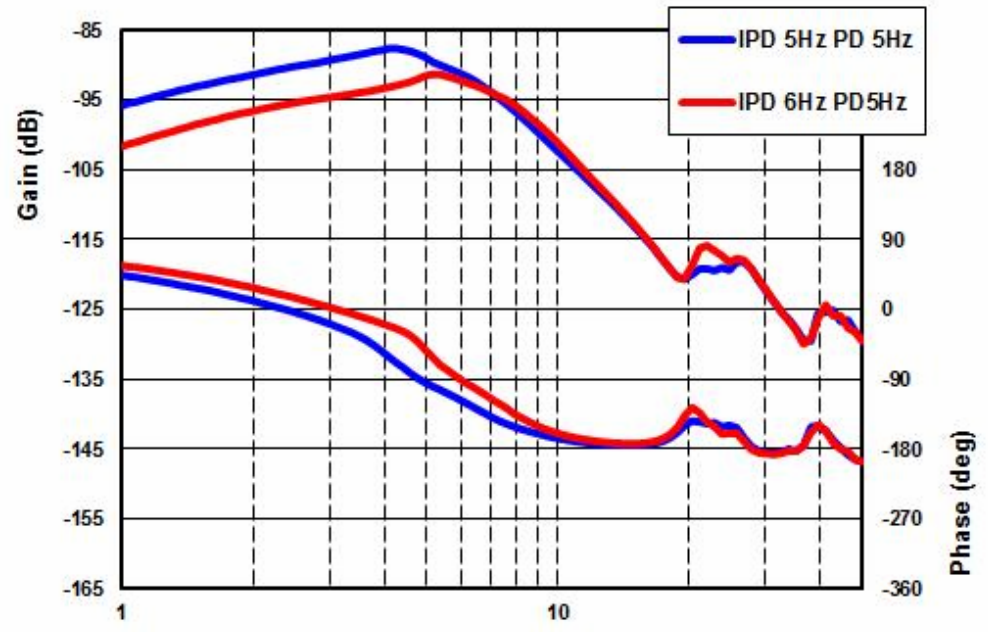

Frequency $(\mathrm{Hz})$

(a) Horizontal translation axis ( $x$-axis)

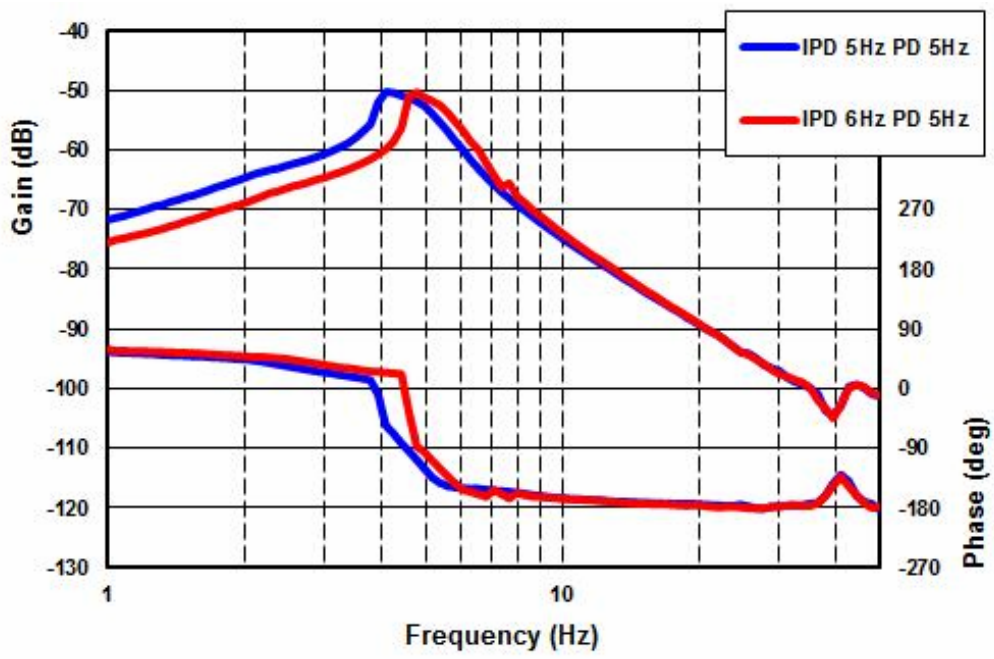

(b) Horizontal rotational axis (yaw-axis)

Fig. 16 Frequency response of the isolation table to direct disturbance $\left(\zeta_{1}=\zeta_{2}=0.5\right)$ 
are seemed to better (smooth) compared to translation axis as the translation motion is subjected to larger sliding contact area as well as higher frictional damping compared to rotational motion although higher rotational $\mathrm{dB}$ gain is recorded in this experiment.

\section{Conclusions}

Disturbance cancellation control technique was implemented to achieve a three-degree-freedom horizontal plane vibration isolation system. The experimental results showed that the system with the proposed controller could realize the zero compliance of the isolation table against static load and provides an effective suppression of dynamic disturbances of the three degree of motions as well. The step responses of the control system demonstrated that the control system might be well stable against both static and dynamic disturbances. High stiffness to the isolation table of the control system against both static and dynamic direct disturbance was also ensured by the experimental results. Vertical supported moving masses of the system are subjected to friction. The induced friction caused adverse effect on the experimental results as well as system performances which will be included in future work.

\section{Acknowledgements}

The authors would like to acknowledge the financial support received from the Ministry of Education, Culture, Sports, Science and Technology of Japan in part as a Grant-in-Aid for Scientific Research (B).

\section{References}

(1) E. I. Rivin, "Vibration Isolation of Precision Equipment", Precision Engineering, vol. 17, pp. 41-56, 1995.

(2) Rivin, E. I., "Passive Vibration Isolation", ASME Press, New York, 2003.

(3) Yasuda, M., Osaka, T., and Ikeda, M., "Feed-forward Control of a Vibration Isolation System for Disturbance Suppression", Proceedings of the $35^{\text {th }}$ Conference on Decision and Control, pp. 1229-1233, 1996.

(4) Fuller, C. R., Elliott, S. J., and Nelson, P. A., "Active Control of Vibration”, Academic Press, pp. 213-220, 1996.

(5) Miyazaki, T., Mizuno, K., Kawatani, R., and Hamada, H., "Consideration about Feedback Feed-forward Hybrid Control for Active Control of Micro-vibration Control", Proceedings of the Second International Conference on Motion and Vibration, pp. 29-34, 1994.

(6) Mizuno, T., Masato Murashita, Takasaki, M. and Ishino, Y., "Pneumatic Three-axis Vibration Isolation System Using Negative Stiffness", Proceedings of the $44^{\text {th }}$ IEEE Conference on Decision and Control, and the European Control conference, Spain, Dec. 2005.

(7) Mizuno, T., Toumiya, T. and Takasaki, M. "Vibration Isolation Using Negative Stiffness" JSME International Journal, Series C, vol. 46, no. 3, 2003.

(8) Takeshi Mizuno, Masaya Takasaki, Daisuke Kishita and Keiichiro Hirakawa, "Vibration Isolation System Combining Zero-Power Magnetic Suspension With Springs", Control Engineering Practice, vol. 15, no. 2, pp. 187-196, 2006.

(9) Hoque, Md. E.,Takasaki, M., Ishino, Y. and Mizuno, T., "Development of a Three-Axis Active Vibration Isolator Using Zero-Power Control", IEEE/ASME Transactions on Mechatronics, vol. 11, no. 4, pp. $462-470$ (2006.08).

(10) Mizuno, T. and Takemori, Y., "A Transfer-Function Approach to the Analysis and Design of Zero-Power Controllers for Magnetic Suspension System" Electrical Engineering in Japan, vol 141, no. 2, pp. 67-75, 2002.

(11) Mizuno, T., Furushima, T., Ishino, Y. and Takasaki, M., "Realization of Zero-Compliance System by Using Displacement Cancellation Control", Journal of Vibration and Control, 16(4): 585-599, 2010.

(12) Shahadat, M. M. Z., "Vibration Isolation System with Displacement Cancellation and Negative Stiffness Control", Master Thesis, Control Engineering Lab., Saitama University, Japan, 2011. 